\title{
Monte Carlo Methods for the Game Kingdomino
}

\author{
Magnus Gedda, Mikael Z. Lagerkvist, and Martin Butler \\ Tomologic AB \\ Stockholm, Sweden \\ Email: firstname.lastname@tomologic.com
}

\begin{abstract}
Kingdomino is introduced as an interesting game for studying game playing: the game is multiplayer (4 independent players per game); it has a limited game depth (13 moves per player); and it has limited but not insignificant interaction among players.

Several strategies based on locally greedy players, Monte Carlo Evaluation (MCE), and Monte Carlo Tree Search (MCTS) are presented with variants. We examine a variation of UCT called progressive win bias and a playout policy (Player-greedy) focused on selecting good moves for the player. A thorough evaluation is done showing how the strategies perform and how to choose parameters given specific time constraints. The evaluation shows that surprisingly MCE is stronger than MCTS for a game like Kingdomino.

All experiments use a cloud-native design, with a game server in a Docker container, and agents communicating using a RESTstyle JSON protocol. This enables a multi-language approach to separating the game state, the strategy implementations, and the coordination layer.

Index Terms-Artificial intelligence, games, Monte Carlo, probabilistic computation, heuristics design.
\end{abstract}

\section{INTRODUCTION}

Implementations and heuristics for computer players in classical board games such as Chess, Go and Othello have been studied extensively in various contexts. These types of games are typically two-player, deterministic, zero sum, perfect information games. Historically, game theoretic approaches such as Minimax and similar variants such as Alpha-Beta pruning have been used for these kinds of games, dating back to Shannon in 1950 [1]. Recently more advanced techniques utilizing Monte Carlo methods [2] have become popular, many of them outperforming the classical game theoretic approaches [3], [4], [5].

The characteristics of the Monte Carlo-based methods also make them viable candidates for games with more complex characteristics such as multiplayer, nondeterministic elements, and hidden information [6]. With the recent emergence of more modern board games (also called eurogames), which often exhibit these characteristics, we naturally see more and more research successfully applying Monte Carlo-based methods to such games [7], [8], [9], [10].

Among the most common Monte Carlo-based methods we have Monte Carlo Evaluation (MCE) (also called flat Monte Carlo) [3] and Monte Carlo Tree Search (MCTS) [11], [12]. Flat Monte Carlo has shown some success [4] but is generally considered too slow for games with deep game trees [13]. MCTS has come to address the problems of MCE and become a popular strategy for modern board games. A plethora of enhancements have been presented for MCTS, both general and domain-dependent, increasing its performance even further for various games [14], [15], [16], [17], [18]. For shallow game trees it is still unclear which Monte Carlo method performs best since available recommendations only concern games with deep trees.

Kingdomino [19] is a new board game which won the prestigious Spiel des Jahres award 2017. Like many other eurogames it has a high branching factor but differs from the general eurogame with its shallow game tree (only 13 rounds). It has frequent elements of nondeterminism and differs from zero sum games in that the choices a player makes generally have limited effect on its opponents. The game state of each round can be quantified to get a good assessment of how well each player is doing which facilitates strong static evaluators. The difference in characteristics compared to previously examined eurogames can potentially render previous recommendations misleading.

We examine static evaluators, Monte Carlo Evaluation (MCE) and Monte Carlo Tree Search using the Upper Confidence Bound for Trees algorithm (UCT). Vanilla implementations of MCE and UCT are compared with various enhancements such as heuristics for more realistic playout simulations and an improvement to UCT which initially steers the selection towards more promising moves. All variants are thoroughly evaluated showing how to select good parameters.

The experimental focus is on heuristic design rather than building efficient competitive agents, i.e., the implementations are meant to be comparative rather than relying on low-level optimization tweaks. All agents are independent processes communicating with a game server using a JSON protocol.

\section{KINGDOMINO}

Kingdomino [19] is a modern board game for 2-4 players released in 2016 where the aim of each player is to expand a kingdom by consecutively placing dominoes provided in a semi-stochastic manner. A domino contains two tiles, each representing a terrain type and can have up to three crowns contributing to the score for its area. The goal is to place the dominoes in a $5 \times 5$ grid with large areas connecting terrains of the same type (using 4-connectivity) containing many crowns to score points.

\section{A. Rules (3-4 Players)}

You begin with your castle tile placed as the starting point of your kingdom and a meeple representing your king. In the first 


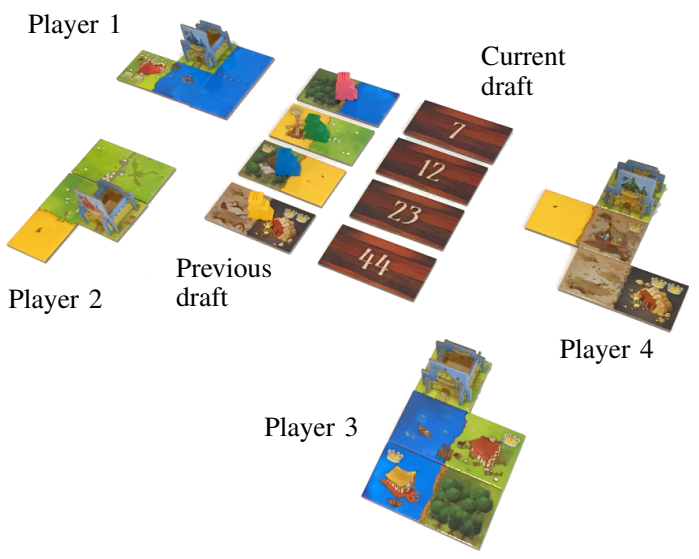

Fig. 1. Kingdomino in-game setup

round, the same number of dominoes as there are kings in play are drawn from the draw pile and added to the current draft. Each player then chooses one domino each from the current draft by placing their king on the chosen domino. When all dominoes in the draft have been chosen, the game moves on to the second round by drawing a new current draft from the draw pile. The previous current draft (the one that now has a king on each domino) becomes the previous draft.

In round two, and every consecutive round up until the last, the player with the king placed on the first domino in the previous draft adds the chosen domino to their territory, according to the connection rules, and chooses a new domino from the current draft by placing the king on the chosen domino. The other players then do the same placementselection move in the order their kings are positioned in the previous draft. A placed domino must either connect to the castle tile or another domino matching at least one of its terrains (horizontally or vertically only). If you cannot add a domino to your kingdom, the domino will be discarded.

The last round works the same as the previous rounds with the exception that there are no more dominoes to draw from the draw pile and therefore there will be no current draft from which to choose any new dominoes.

The final score is the sum of the scores for each 4-connected area of the same terrain type. The score for each area is the number of tiles multiplied by the total number of crowns on the area. Note that for an area with no crowns, the score is zero. There are also two additional rules used in this paper (both part of the official game rules). The first is the Middle Kingdom rule, which states that you get an additional 10 points if your castle is in the center of the $5 \times 5$ grid. The second is the Harmony rule, which states that you get an additional 5 points if your territory is complete (i.e., no discarded dominoes).

For a complete description of the rules, including rules for 2 players, we refer to [19].

\section{B. Game characteristics}

Kingdomino is classified as a non-deterministic game since the dominoes are drawn randomly from the draw pile. All

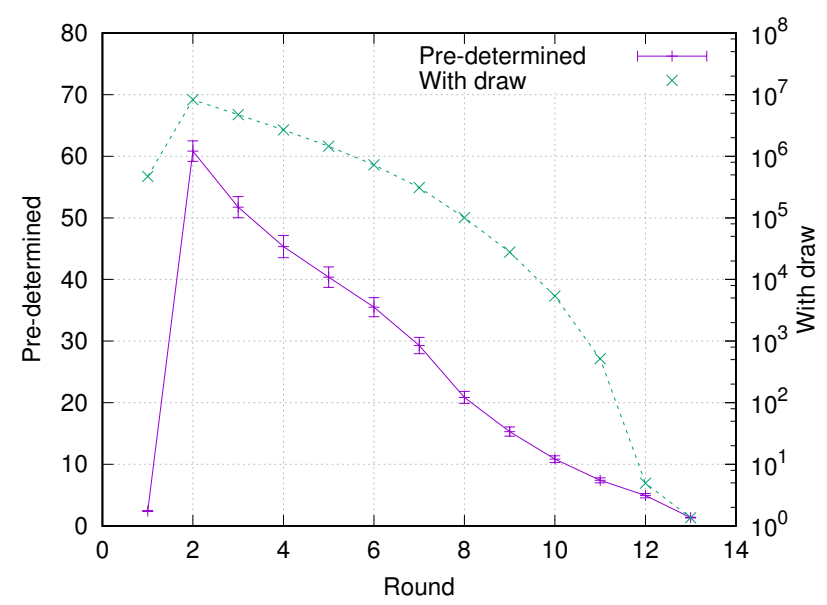

Fig. 2. Average branching factor per round for a random player when playing against three random opponents (1000 games). The error bars show the $95 \%$ confidence interval.

players have a similar goal and all players have complete information of the game state at all times, which means that it is also a symmetric perfect information game.

The number of possible draws from the deck is defined by the following formula. $\prod_{i=0}^{11}\left(\begin{array}{c}48-4 i \\ 4\end{array}\right) \approx 3.4 \cdot 10^{44}$ The most interesting thing about the number of possible draws is that it is significantly less than the total number of shuffles of the deck (around a factor of $3.6 \cdot 10^{16}$ ).

Fig. 2 shows the branching factor for each round. This is computed experimentally using 4-player games with the players choosing moves randomly (see Section V-A. Assuming that the branching factor for player $p$ in round $r$ is an independent stochastic variable $B_{p r}$, multiplying the expected value for the branching factor each round gives the expected value for the game tree size given a predetermined deck shuffle. Using the experimentally determined values for $B_{p r}$, the game tree size is approximately

$$
E\left[\prod_{p=1}^{4} \prod_{r=1}^{13} B_{p r}\right]=\prod_{p=1}^{4} \prod_{r=1}^{13} E\left[B_{p r}\right] \approx 3.74 \cdot 10^{61}
$$

When accounting for the number of possible draws from the deck, the number of Kingdomino games is around $1.27 \cdot 10^{106}$. This puts Kingdomino at a game tree complexity between Hex and Chess when accounting for all shuffles, and similar to Reversi/Othello for a pre-determined shuffle [20].

\section{StRATEgies FOR Kingdomino}

Agents can be implemented using a wide range of strategies. Here we focus on statistical evaluators such as Monte Carlo Evaluation and Monte Carlo Tree Search together with various enhancements. We also include some static evaluators to analyse game characteristics and use as reference agents when evaluating the statistical strategies.

\section{A. Static Evaluators}

Kingdomino generally has a solid score progression which makes it feasible to implement strong static evaluators by 
computing the score of each player at every state of the game, unlike, e.g., the game of Go which has to rely heavily on statistical methods since domain-dependent move generators are very difficult to improve [4]. Also, considering Kingdomino is a perfect information game, any static evaluator with a greedy approach could potentially be competitive. We define two static evaluators, Greedy Placement Random Draft (GPRD) and Full Greedy (FG). GPRD places each domino in a greedy manner (to get maximum point increase) but selects dominoes randomly from the current draft while FG uses both greedy placement and selects greedily from the current draft. Both evaluators avoid moves that break the Middle Kingdom rule or result in single-tile holes. The FG evaluator is likely to act similar to an above average human player since it incorporates the visible domain knowledge to make realistic moves without using any search strategies.

\section{B. Monte Carlo Methods}

Monte Carlo methods such as Monte Carlo Evaluation (MCE) [3] and Monte Carlo Tree Search (MCTS) [11], [12] have recently been used successfully for building computer players in both classical two-player deterministic board games, such as Go [4], and more modern multiplayer nondeterministic board games, such as Settlers of Catan [7], Scotland Yard [8], and 7 Wonders [9].

1) Monte Carlo Evaluation: In flat Monte Carlo search (which we in this paper refer to as Monte Carlo Evaluation), each game state is represented by a node in a tree structure and the edges represent possible moves. The root node represents the current game state and its children represent the game states produced by each available move. The evaluation selects a child node randomly (using uniform sampling) and simulates a complete game from that node (referred to as a playout), using some playout policy, until termination. The selectionplayout procedure is done repeatedly until either a maximum number of playouts have been reached or the time runs out. Each child node stores the average result from all its playouts, and the the max child is selected as the best move. Evaluators based on MCE have shown to be strong players in small classical games, such as $3 \times 3$ Tic-Tac-Toe, and play on par with standard evaluators on larger games [3].

The high exponential cost of searching trees with high branching factors makes global tree search impossible, especially under tight time constraints. However, the search depth of Kingdomino is shallow enough for MCE to potentially be a viable option since a shallow game tree facilitates high termination frequencies even at early stages in the game.

2) Monte Carlo Tree Search: Monte Carlo Tree Search expands on the functionality of Monte Carlo Evaluation by expanding the search tree asymmetrically in a best-first manner guided by statistics. A commonly used Monte Carlo Tree search algorithm for game play is UCT [11], which guides the search by computing the Upper Confidence Bound (UCB) for each node and select moves for which the UCB is maximal.
The UCB is defined as

$$
\mathrm{UCB}=\bar{X}_{i}+C \sqrt{\frac{\ln T}{T_{i}}},
$$

where $\bar{X}_{i}$ is the average payoff of move $i, T$ is the number of times the parent of $i$ has been visited, $T_{i}$ is the number of times $i$ has been sampled, and $C$ is the exploration constant. For a full description of the UCT algorithm we refer to [11]. UCT, with enhancements such as domain-specific heuristics in the playout policies, has been shown to perform well for games with high branching factors [6].

\section{Playout Policy Enhancements}

The playout policy in its standard form uses random move selection throughout the playout. A common enhancement is to incorporate, potentially time expensive, domain-dependent heuristics to get more realistic playouts. We examine four different playout policies. The true random playout policy (TR) which chooses all moves randomly in the playout. The $\epsilon$-greedy policy $(\epsilon \mathrm{G})[6]$ which chooses moves randomly with $\epsilon$ probability and greedily with probability $(1-\epsilon)$. The full greedy policy (FG) which chooses all moves greedily. And finally we use a playout policy we call the player-greedy policy (PG). It chooses the player's move greedily and all opponent moves randomly. Random opponent modelling has recently been applied successfully in multi-player tracks of General Video Game Playing (GVGP) AI competitions [21] but has, to our knowledge, not previously been applied to AI in board games. The player-greedy policy should be favourable in Kingdomino since the actions of the opponents generally have limited (but not insignificant) impact on the player. Its success in the GVGP setting can likely be attributed to the tight time constraints for opponent modelling in GVGP.

The $\epsilon$-greedy and player-greedy strategies combine the advantage of domain knowledge with the speed provided by random move selection. With a low branching factor, there is a reasonable chance that good moves will be made with some frequency in random sampling. But games with large branching factors, such as Kingdomino, generally have many irrelevant, or even detrimental, moves. In these games the probability of playing out good moves during random playouts is relatively small, so there should be a large benefit to using informed simulation strategies.

\section{Scoring Functions}

The scoring function defines how the result of a playout is measured. The basic scoring function is the Win Draw Loss function (WDL) which simply gives a winning playout the score 1, a playout where the player is tied with an opponent for first place (a draw) the score 0.5 , and a playout which is not a win or a draw the score 0 . The reward model in Monte Carlo Evaluation facilitates more sophisticated scoring functions. One such function, which we refer to as the Relative scoring function $(\mathrm{R})$, takes the player's score relative to the score of the highest scoring opponent $f=p_{s} /\left(p_{s}+q_{s}\right)$, where $p_{s}$ is the player score and $q_{s}$ is the opponent score. A third 
third scoring function, which we refer to as the Player scoring function $(\mathrm{P})$, simply uses the player's score. This function does not care whether the player wins or loses and only tries to maximize the player's own score.

\section{E. MCTS Selection Enhancements}

Among the popular enhancements for MCTS there are learning enhancements such as RAVE [16] and the history heuristic [14], [15]. They use offline information from previous games to guide the selection toward moves that have been successful in past games. Kingdomino has a low $n$-ply variance which means it could potentially benefit from learning enhancements [6]. However, in Kingdomino the reward of a single move is dependent on the game state, so the game state has to be incorporated in the offline information for each move. This has the effect of drastically decreasing the hit probability of a move while increasing lookup time.

A popular online enhancement is progressive bias [17] which guides the selection towards promising moves by using a - potentially time consuming - heuristic value which diminishes with increasing visits to the node. Here we use a selection enhancement which we call progressive win bias which combines progressive bias with a tweak that makes the heuristic value diminish with the number of node losses instead of the number of node visits. The tweak has successively been applied to the game Lines of Action [22] but has never been evaluated in a systematic fashion as presented here. We define progressive win bias as

$$
W \frac{H_{i}}{T_{i}\left(1-\bar{X}_{i}\right)+1},
$$

where $H_{i}$ is the heuristic value, $\bar{X}_{i}$ is the average reward for the node, $T_{i}$ is the number of node visits, and $W$ is a positive constant which controls the impact of the bias. In this paper we use $H_{i}=S_{i}-S_{i-1}$ as heuristic, where $S_{\gamma}$ is the player's score after move $\gamma$. The formula is simply added to the regular $\mathrm{UCB}$ in 1

\section{IMPLEMENTATION}

The implementation for the game is based on a server-client architecture. The server maintains all current, future, and past games, while a client agent can play in one or more games. A game is initiated with a set number of players, putting it in the list of future games. An agent can join a game, on which it receives a secret token enabling it to make moves for a player in the game. After enough players join the game, it is started. The game server has a graphical front-end showing all current and past games with full history for analysis and inspection.

Agents poll the server for the current game state: the kingdoms and their scores; the current and next draft; the current player; all possible moves; and all previously used dominoes. To make a move, the agent for the current player chooses one of the possible moves. The communication is based on a HTTP REST JSON API. The protocol gives enough information to enable stateless agents that only need remember their secret token. When joining a game, it is possible for an agent to register an HTTP callback endpoint that the server uses to notify the agent that its player is the current player.

The game server is implemented in Scala, and is packaged as a Docker container. This simplifies running the server in any setting, either on a remote server or locally. In particular, the choice of using standard web technologies for communication leads to a clean and simple separation of agents and the server.

At a one-day hackathon, 7 programmers could without preparation build rudimentary game playing agents in a variety of languages (Java, Scala, Python, Rust, and Haskell). The state representation and the full valid move list make it simple to implement static evaluators, without having to implement the full game logic. Naturally, for a more competitive client the full game logic needs to be implemented also in the client.

\section{EXPERIMENTS}

Our experiments are intended to give insights into the game, to give guidance on what strategies and algorithms are useful, and how to tune parameters for the strategies. To compare strategies, we have made the choice to use static time limits per ply to study how well different strategies can make use of a specific time allotment without introducing the complexities of full time management.

Note that all games in these experiments are 4-player games (unless otherwise stated), so a when a strategy plays equally well as its opponent it will result in a $25 \%$ win rate. All intervals (in both figures and tables) represent the $95 \%$ confidence interval.

In board games the number of victories alone can be considered insufficient to determine the strength of a player. This is supported by the USOA (United States Othello Association) which uses the margin of victory as the single most important feature in determining a player's rating [3]. Therefore, most of our experiments use the victory margin to determine player strength.

\section{A. Setup}

All agents used in the experiments are written in Java and run on a single threaded $3.2 \mathrm{GHz}$ Intel Core i7 with $12 \mathrm{~GB}$ RAM that is also running the game server. While the agents are not written to be the fastest possible, some care has been taken to keep the implementation reasonably fast. The goal is to facilitate comparison between the agents, not to implement a certain algorithm optimally.

\section{B. Agents}

We use three different static evaluator agents: the True Random (TR) agent, the Greedy Placement Random Draft (GPRD) agent, and the Full Greedy (FG) agent. The FG agent is used as reference player against which we evaluate all statistical players.

Each Monte Carlo Evaluation agent is implemented using flat Monte Carlo search and characterized by a playout policy/scoring function combination. We denote them by MCE$X / Y$ where $X$ is the playout policy and $Y$ is the scoring function. 


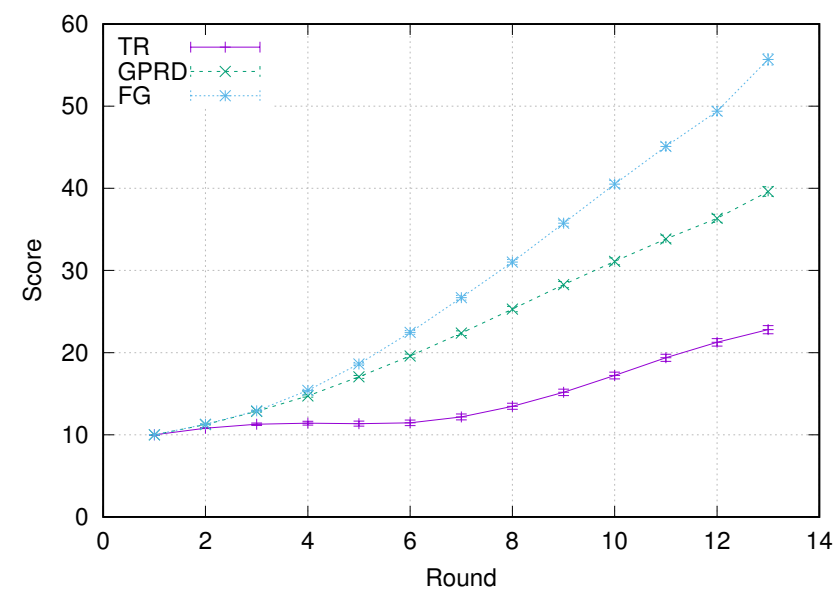

Fig. 3. Average scores against three TR opponents (1000 games).

The Monte Carlo Tree Search agents all use the WDL scoring function and are therefore only characterized by playout policy and selection enhancements. The MCTS agents lack the possibility of using a relative scoring function but use maximum score increase as tie breaker for moves of equal win rate. We denote the standard MCTS agents by UCT- $X$, the MCTS agents using progressive bias by $\mathrm{UCT}_{\mathrm{B}}-X$, and progressive win bias by $\mathrm{UCT}_{\mathrm{W}}-X$, where $X$ is the playout policy.

\section{Impact of Domain Knowledge}

In the first experiment we wanted to quantify how basic domain knowledge affects strategies based on static evaluators. We did this by playing a True Random player (TR), a Greedy Placement Random Draft player (GPRD), and a Full Greedy player (FG) 1000 games each against three TR opponents and registered the number of wins, draws, and losses. We also registered the score after each round in every game to see the general score progression of each strategy.

The average score progression for the three different strategies over is shown in Fig. 3 All players start with 10p since the castle is within three tiles distance from the tile furthest away, thus fulfilling the Middle Kingdom rule. We can clearly see that the TR player had trouble increasing its score and even dipped around Round 5-6 due to breaking the Middle Kingdom rule. The GPRD player did a better job, showing that it is of great importance to select good positions for the placed domino. However, the score progression of the FG player indicates that it is of equal importance to also select a good domino from the current draft (the score for FG is approximately twice the score of GPRD when corrected for the scores of random moves).

The number of wins, losses, and draws for each strategy are shown in Table I Here we see that the FG player truly outplayed the TR opponents, which was anticipated. More interesting is that the GPRD player only has approximately $79 \%$ win rate against the TR opponents. So while carefully selecting placements, making an uninformed selection from

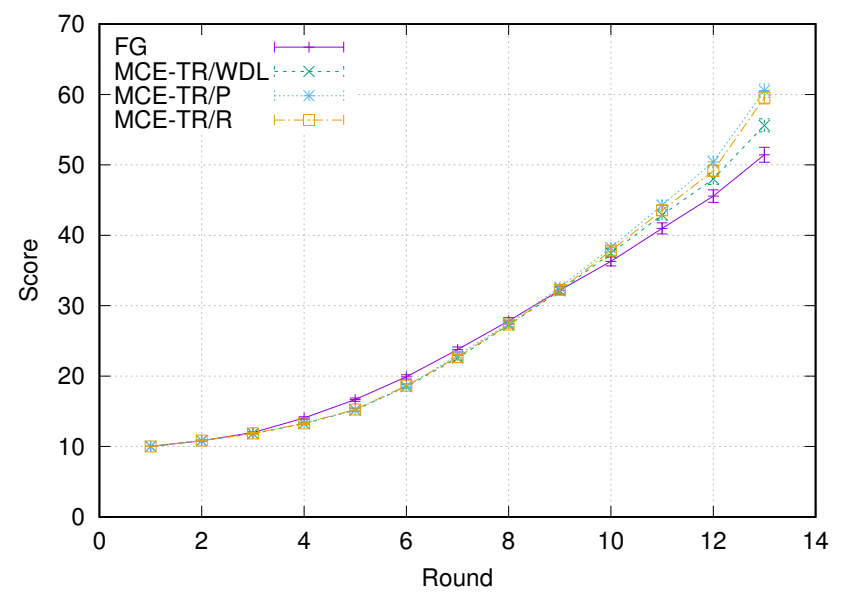

Fig. 4. Average scores against three FG opponents (500 games).

TABLE I

WIN PERCENTAGES FOR 1000 GAMES AGAINST THREE TR OPPONENTS.

\begin{tabular}{lrrr}
\hline Player Strategy & \multicolumn{3}{c}{ Opponent Strategy } \\
& \multicolumn{3}{c}{ TR } \\
\cline { 2 - 4 } & Wins (\%) & Draws $(\%)$ & Losses $(\%)$ \\
\hline TR & $223(22.3)$ & $29(2.9)$ & $748(74.8)$ \\
GPRD & $794(79.4)$ & $22(2.2)$ & $184(18.4)$ \\
FG & $977(97.7)$ & $2(0.2)$ & $21(2.1)$ \\
\hline
\end{tabular}

the current draft has a noticeable impact when played against random opponents.

\section{Static vs Statistical Evaluators}

In this experiment we investigated how simple statistical evaluation performs compared to the best static evaluatorbased strategy. We also look at how different scoring functions affect the performance of the statistical evaluators. We did this by playing three Monte Carlo Evaluation players, each using a different scoring function and random selection playout policy, 500 games each against three FG opponents and compared the results to the same number of games played by a FG player against three FG opponents. The time limit was set to $5 \mathrm{~s}$ per ply. The three Monte Carlo players were MCETR/WDL, which only counts the number of wins/draws/losses and chooses the move that maximises the number of wins, MCE-TR/P, which tries to maximise the player's final score, and MCE-TR/R, which tries to maximise the victory margin. The score progressions are shown in Fig. 4 and the final scores in Table [I]

The experiment clearly shows that the statistical evaluators significantly outperform the FG player. It is interesting to see how the statistical evaluators select sub-greedy moves in the middle of the game to enable higher payoffs in the later parts of the game. It is also clear that MCE-TR/WDL does not reach as high final score as the other statistical evaluators. This is most likely a result of the WDL scoring function's lack of score information which renders it incapable of discriminating 
TABLE II

AVERAGE SCORES FOR 500 GAMES AGAINST THREE FG OPPONENTS.

\begin{tabular}{ll}
\hline Player Strategy & Avg. Score \\
\hline FG & $51.4(2.1)$ \\
MCE-TR/WDL & $55.6(1.8)$ \\
MCE-TR/P & $60.6(1.9)$ \\
MCE-TR/R & $59.5(1.8)$ \\
\hline
\end{tabular}

between branches where all leaf nodes result in a win while it is in the lead. Since each node only stores the winning average, it will not be able to determine which branch will lead to a higher final score. Also, the $\mathrm{R}$ and $\mathrm{P}$ scoring functions are more robust against the recurring stochastic events. There is no significant difference in performance between the Player scoring function and Relative scoring function.

\section{E. Enhanced Playout Policies}

In this experiment we investigated the effect of different enhancements to Monte Carlo Evaluation by incorporating domain knowledge into the playout policies. We did this by playing Monte Carlo Evaluation players, both with and without domain knowledge, against three FG opponents and compared the results. The players we used were MCE-TR/R, which has no domain knowledge at all and only selects moves randomly for both the player and opponents in the playouts, MCE$\epsilon \mathrm{G} / \mathrm{R}$ with $\epsilon=0.75$, which uses random selection in $75 \%$ of the times in the playout and greedy selection $25 \%$ of the times, MCE-PG/R, which uses greedy selection for the player and random selection for the opponents in the playouts, and MCE-FG/R, which uses greedy selection for all moves in the playouts. We used the relative scoring function since its goal aligns with the measure of player strength and facilitates easier analysis of the result plots.

Since all games in the experiment were 4-player games and $\epsilon$ was set so that greedy selection will be used $25 \%$ of the time, the number of greedy move evaluations would be the same for both MCE- $\epsilon \mathrm{G} / \mathrm{R}$ and MCE-PG/R and should result in approximately the same playout frequency for the two simulation strategies. This will tell us how important accurate opponent modelling is in Kingdomino.

Fig. 5 shows the victory margin under various time constraints for the different strategies (each point represents 200 games). In addition to the Monte Carlo Evaluation game strategies, the result from playing 200 games with an FG player against three FG opponents is also shown (the solid red line with the $95 \%$ confidence interval as dotted red lines). Fig. 6 shows the number of playouts per second for each playout policy.

The experiment shows that the FG evaluator is competitive to the statistical evaluators under tight time constraints. It is comparable to MCE-TR/R, and outperforms all the others, when the time is capped to $0.1 \mathrm{~s}$ per move. It also shows that the best knowledge-based statistical evaluators need approximately $0.5-1 s$ time per move for the extra heuristic computations to pay off compared to selecting playout moves

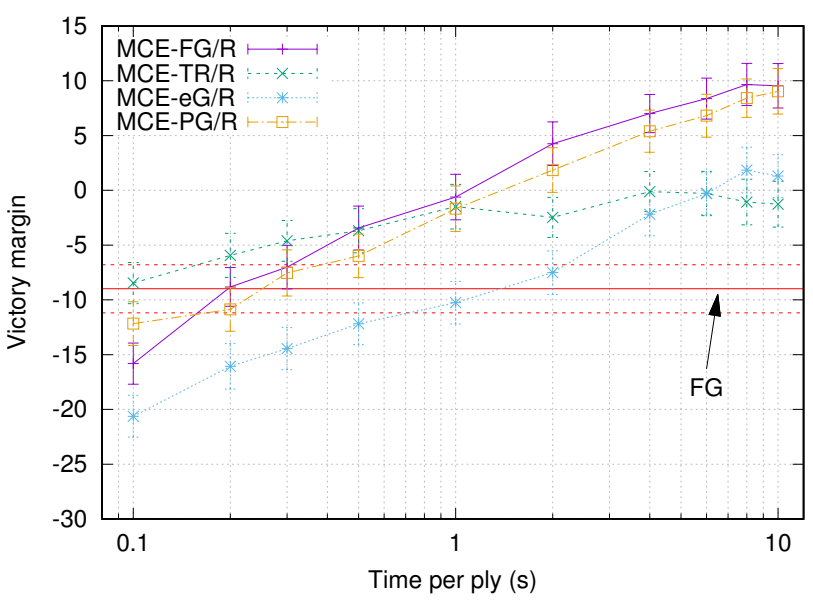

Fig. 5. Average victory margins against three FG opponents.

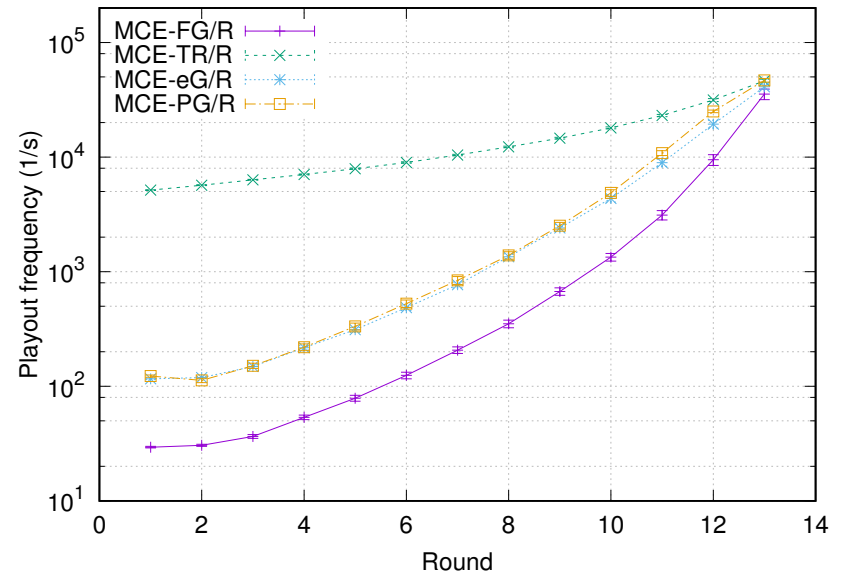

Fig. 6. Average playout frequency (200 games).

randomly, but they consistently outperform the random playout policy for move times $>1 s$. It also shows that it is more important to model the player's own move realistically than the moves of the opponent. This is clear from the difference in performance between MCE-PG/R and MCE- $\epsilon \mathrm{G} / \mathrm{R}$ when having approximately the same playout frequencies. Furthermore, if we compare MCE-PG/R to MCE-FG/R we see that realistic opponent modelling is disadvantageous for short ply times $(<0.2 s)$. This is natural since realistic opponent modelling is costly and MCE-FG/R will only have time for few playouts before selecting its move, while MCE-PG/R can produce more playouts and have a better statistical sample when choosing its move. However, once the number of playouts go up $(>0.1 s)$ we see that realistic opponent modelling consistently outperforms the player-greedy strategy, although not by much.

\section{F. Tree Search}

We examined the UCB exploration constant $C$ by playing an UCT-TR and an UCT-FG player against three FG players for various values of $C$. The result is shown in Fig. 77. The experiment shows that $C=0.6$ is a suitable value for players 


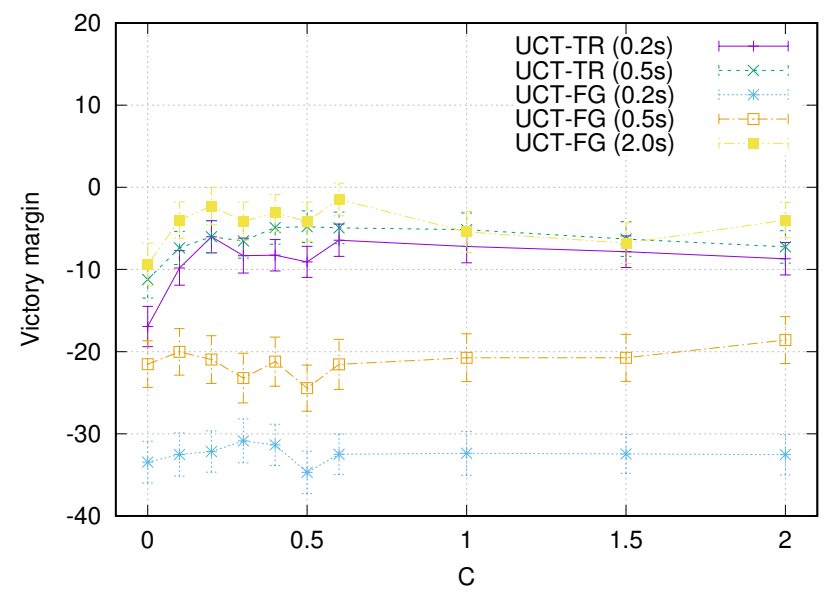

Fig. 7. Average victory margins against three FG opponents.

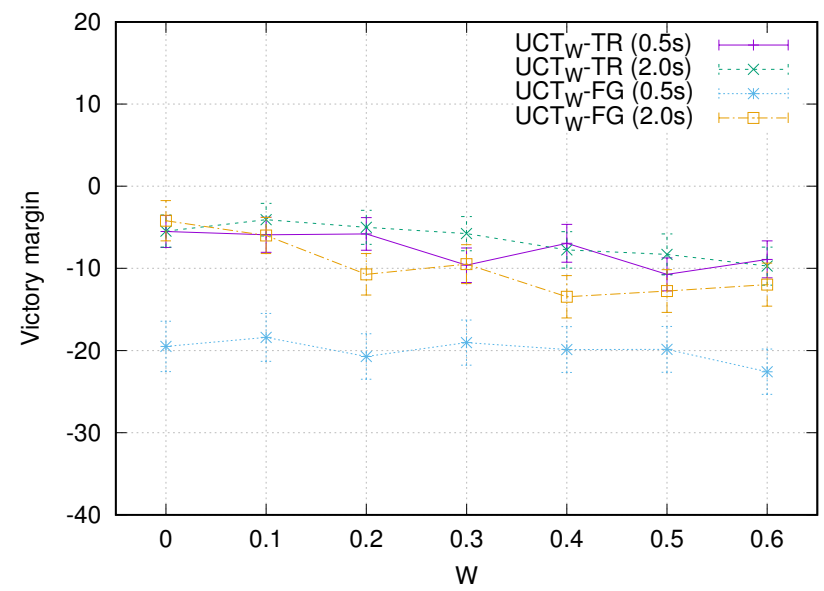

Fig. 8. Average victory margins against three FG opponents.

with many playouts per ply and $C \geq 1.0$ for strategies with few playouts per ply. A theory is that due to Kingdomino's frequent stochastic events, a move requires numerous playouts to accumulate a representative reward. So there is a risk of focusing the tree expansion on high-reward moves before all moves get representative rewards. Therefore, players with few playouts per ply should perform better with a higher exploration constant.

We also examined the impact constant $W$ for progressive bias and progressive win bias by playing a $\mathrm{UCT}_{\mathrm{W}}$-TR player and a $\mathrm{UCT}_{\mathrm{W}}-\mathrm{FG}$ player, both with $C=0.6$, against three $\mathrm{FG}$ opponents for various values of $W$. The result is shown in Fig. 8 It shows that we get the highest performance impact for $W=0.1 \sim 0.2$ and after that the performance decreases with $W$.

\section{G. Comparing Strategies}

Table III shows the performance of all strategies for 200 games played against three FG opponents. The 95\% confidence intervals are in the range $[3.5,6.0]$ for all entries, with the majority near the lower limit. The highest performer for each time constraint is marked by a dark blue box. Performances within $5 \%(10 \%)$ of the best are marked by a light (lighter) blue box. The UCB exploration constant was set to $C=0.6$ for all UCT strategies and the the bias impact factor was set to $W=0.1$ for $\mathrm{UCT}_{\mathrm{B}}{ }^{*}$ and $\mathrm{UCT}_{\mathrm{W}}{ }^{*}$.

The results show that for each time constraint the best MCE variant consistently outperforms all variants of UCT. A possible theory is that UCT is hampered by its WDL scoring function, but further experiments verifying this hypothesis is outside the scope of this paper. The true random playout policy variant $(\mathrm{MCE}-\mathrm{TR} / \mathrm{R})$ excels for short ply times $t<0.5 \mathrm{~s}$. After that the full greedy playout policy variant (MCE-FG/R) gets enough time each ply to produce rewards representative enough to reliably select trajectories in the game tree that outperform the the random playout policy, in spite of the significantly higher playout frequency of the random playout policy. The MCE-PG performs almost on par with MCE-FG which indicates that allocating time for accurate opponent modelling only has a small gain compared to using random move selection for the opponents.

The results also show that the UCT enhancements improve the results for tight time constraints $(t<0.2 s)$, which is expected due to few playouts, but are otherwise on par with regular UCT.

\section{CONClusions AND Future Work}

This paper introduces Kingdomino as an interesting game to study for game playing. The shallow game tree and relatively limited interaction between players of Kingdomino combined with the stochastic nature and possibility to evaluate partial game states is particularly interesting. The results indicate that for games such as Kingdomino, MCE is superior to UCT, which would infer new recommendations on the suitability of MCE for games of similar complexity. This is especially interesting, given that an MCE evaluator is significantly easier to implement correctly and efficiently than full UCT.

The player-greedy playout policy is surprisingly effective, balancing exploration power with (expensive) local evaluation. Our belief is that this is due to the limited (but not insignificant) interaction among players in Kingdomino, but further experiments in other games are needed to verify this hypothesis. The progressive win bias selection improvement shows promise as a way to combine a heuristic evaluation with the current knowledge gained from the exploration, but further experiments in other settings better suited for the UCT is needed to analyse its impact.

Our evaluation uses thorough systematic examination of all constants involved to avoid the presence of magic numbers which frequently occur without explanation in many similar papers in the field. It also uses new and illuminating graphs for showing the impact of different choices. In particular, the usage of victory margin in favour of win percentages is very powerful for a multi player score maximization game such as Kingdomino. These graphs have helped us gain new insights into both the game and how our strategies perform. 
TABLE III

AVERAGE VICTORY MARGINS FOR 200 GAMES AGAINST THREE FG OPPONENTS.

\begin{tabular}{|c|c|c|c|c|c|c|c|c|c|c|}
\hline \multirow[t]{2}{*}{ Strategy } & \multicolumn{10}{|c|}{ Time per ply } \\
\hline & $0.1 \mathrm{~s}$ & $0.2 \mathrm{~s}$ & $0.3 \mathrm{~s}$ & $0.5 \mathrm{~s}$ & $1.0 \mathrm{~s}$ & $2.0 \mathrm{~s}$ & $4.0 \mathrm{~s}$ & $6.0 \mathrm{~s}$ & $8.0 \mathrm{~s}$ & $10.0 \mathrm{~s}$ \\
\hline FG & -9.0 & -9.0 & -9.0 & -9.0 & -9.0 & -9.0 & -9.0 & -9.0 & -9.0 & -9.0 \\
\hline MCE-TR/R & -8.5 & -5.9 & -4.6 & -3.7 & -1.5 & -2.5 & -0.1 & -0.3 & -1.1 & -1.3 \\
\hline MCE-FG/R & -15.8 & -8.8 & -7.0 & -3.4 & -0.6 & 4.3 & 7.0 & 8.4 & 9.7 & 9.6 \\
\hline MCE-PG/R & -12.2 & -10.9 & -7.5 & -6.0 & -1.7 & 1.9 & 5.4 & 6.8 & 8.4 & 9.0 \\
\hline MCE- $\epsilon \mathrm{G} / \mathrm{R}$ & -20.6 & -16.0 & -14.5 & -12.2 & -10.3 & -7.5 & -2.2 & -0.3 & 1.9 & 1.3 \\
\hline UCT-TR & -13.5 & -6.4 & -7.3 & -4.9 & -5.5 & -4.4 & -3.5 & -5.2 & -7.2 & -4.4 \\
\hline UCT-FG & -38.3 & -32.5 & -29.4 & -21.6 & -12.0 & -1.5 & -0.2 & 3.5 & 4.0 & 3.9 \\
\hline UCT-PG & -25.8 & -20.7 & -15.5 & -15.3 & -13.9 & -10.4 & -7.1 & -7.4 & -6.2 & -4.1 \\
\hline UCT $-\epsilon \mathrm{G}$ & -33.3 & -24.0 & -16.2 & -15.7 & -9.0 & -7.0 & -3.1 & -4.0 & -2.6 & -1.3 \\
\hline $\mathrm{UCT}_{\mathrm{B}}-\mathrm{TR}$ & -10.1 & -7.4 & -6.1 & -7.9 & -4.6 & -6.7 & -4.8 & -4.3 & -2.9 & -4.5 \\
\hline $\mathrm{UCT}_{\mathrm{B}}-\mathrm{FG}$ & -39.8 & -30.6 & -29.7 & -21.6 & -11.7 & -5.9 & -0.1 & 3.2 & 3.2 & 1.4 \\
\hline $\mathrm{UCT}_{\mathrm{B}}-\mathrm{PG}$ & -25.5 & -20.8 & -19.7 & -15.4 & -13.4 & -9.1 & -7.6 & -6.3 & -4.5 & -12.4 \\
\hline $\mathrm{UCT}_{\mathrm{B}}-\epsilon \mathrm{G}$ & -31.5 & -25.2 & -20.2 & -16.3 & -10.7 & -8.1 & -4.1 & -2.6 & -1.9 & -2.9 \\
\hline $\mathrm{UCT}_{\mathrm{W}}-\mathrm{TR}$ & -11.4 & -6.7 & -7.3 & -5.9 & -4.6 & -4.1 & -5.8 & -5.0 & -4.0 & -4.5 \\
\hline $\mathrm{UCT}_{\mathrm{W}}-\mathrm{FG}$ & -42.6 & -33.0 & -30.3 & -18.4 & -13.9 & -6.0 & -2.5 & 0.6 & 1.2 & 1.4 \\
\hline $\mathrm{UCT}_{\mathrm{W}}-\mathrm{PG}$ & -29.2 & -24.3 & -20.0 & -19.7 & -15.4 & -16.9 & -13.1 & -12.2 & -13.9 & -12.4 \\
\hline $\mathrm{UCT}_{\mathrm{W}}-\epsilon \mathrm{G}$ & -30.5 & -23.0 & -22.8 & -16.6 & -13.2 & -6.6 & -5.4 & -3.1 & -2.7 & -2.9 \\
\hline
\end{tabular}

For future work one MCTS enhancement alternative could be a learning heuristic that keep offline information on the success of placement positions for different kingdom patterns. Experienced human players tend to place dominos in a structured pattern to avoid single tile holes in the kingdom. It would also be interesting to implement agents using completely different strategies such as deep reinforcement learning.

The code for the Kingdomino game server can be downloaded from https://github.com/mratin/kdom-ai, and the AI implementations can be downloaded from https://github.com/mgedda/kdom-ai.

\section{ACKNOWLEDGEMENTS}

We thank all participants at Tomologic who implemented agents and discussed strategies with us.

\section{REFERENCES}

[1] Shannon, C.E.: XXII. Programming a computer for playing chess. The London, Edinburgh, and Dublin Philosophical Magazine and Journal of Science 41(314) (1950) 256-275

[2] Metropolis, N., Ulam, S.: The monte carlo method. Journal of the American statistical association 44(247) (1949) 335-341

[3] Abramson, B.: Expected-outcome: A general model of static evaluation. IEEE TPAMI 12(2) (1990) 182-193

[4] Bouzy, B., Helmstetter, B.: Monte-carlo Go developments. In: Advances in computer games. Springer (2004) 159-174

[5] Silver, D., Huang, A., Maddison, C.J., Guez, A., Sifre, L., Van Den Driessche, G., Schrittwieser, J., Antonoglou, I., Panneershelvam, V., Lanctot, M., et al.: Mastering the game of Go with deep neural networks and tree search. Nature 529(7587) (2016) 484-489

[6] Sturtevant, N.R.: An analysis of UCT in multi-player games. In: Intern. Conference on Computers and Games, Springer (2008) 37-49

[7] Szita, I., Chaslot, G., Spronck, P.: Monte-carlo tree search in Settlers of Catan. In: Advances in Computer Games, Springer (2009) 21-32

[8] Nijssen, J.P.A.M., Winands, M.H.M.: Monte carlo tree search for the hide-and-seek game Scotland Yard. IEEE Transactions on Computational Intelligence and AI in Games 4(4) (2012) 282-294

[9] Robilliard, D., Fonlupt, C., Teytaud, F.: Monte-carlo tree search for the game of "7 Wonders". In: Workshop on Computer Games, Springer (2014) 64-77
[10] Browne, C.B., Powley, E., Whitehouse, D., Lucas, S.M., Cowling, P.I., Rohlfshagen, P., Tavener, S., Perez, D., Samothrakis, S., Colton, S.: A survey of monte carlo tree search methods. IEEE Transactions on Computational Intelligence and AI in games 4(1) (2012) 1-43

[11] Kocsis, L., Szepesvári, C.: Bandit based monte-carlo planning. In: European conference on machine learning, Springer (2006) 282-293

[12] Coulom, R.: Efficient selectivity and backup operators in monte-carlo tree search. In: International conference on computers and games, Springer (2006) 72-83

[13] Nijssen, J.P.A.M.: Monte-Carlo Tree Search for Multi-Player Games. PhD thesis, Maastricht University, The Netherlands (12 2013)

[14] Schaeffer, J.: The history heuristic. ICCA Journal 6(3) (1983) 16-19

[15] Winands, M.H.M., van der Werf, E.C.D., van den Herik, H.J., Uiterwijk, J.W.H.M.: The relative history heuristic. In: International Conference on Computers and Games, Springer (2004) 262-272

[16] Gelly, S., Silver, D.: Combining online and offline knowledge in UCT. In: Proceedings of the 24th international conference on Machine learning, ACM (2007) 273-280

[17] Chaslot, G.M.J.B., Winands, M.H.M., Herik, H.J.v.d., Uiterwijk, J.W.H.M., Bouzy, B.: Progressive strategies for monte-carlo tree search. New Mathematics and Natural Computation 4(03) (2008) 343-357

[18] Nijssen, J.P.A.M., Winands, M.H.M.: Enhancements for multi-player monte-carlo tree search. In: International Conference on Computers and Games, Springer (2010) 238-249

[19] Cathala, B., Bouquet, C.: Kingdomino (2016)

[20] Wikipedia contributors: Game complexity - Wikipedia, the free encyclopedia (2018) [Online; accessed 2018-02-15].

[21] Gaina, R.D., Couëtoux, A., Soemers, D.J.N.J., Winands, M.H.M., Vodopivec, T., Kirchgeßner, F., Liu, J., Lucas, S.M., Perez-Liebana, D.: The 2016 two-player gvgai competition. IEEE Transactions on Computational Intelligence and AI in Games (2017)

[22] Winands, M.H.M., Bjornsson, Y., Saito, J.T.: Monte carlo tree search in lines of action. IEEE Transactions on Computational Intelligence and AI in Games 2(4) (2010) 239-250 